\title{
SEASONAL SYMPTOM VARIATION IN PATIENTS WITH CHRONIC FATIGUE: COMPARISON WITH MAJOR MOOD DISORDERS
}

\author{
Jon Kar Zubieta, ${ }^{*}$ N. Cary Engleberg, $†$ L. Ilhan Yargiç, $\ddagger$ Atul C. Pande, ${ }^{*}$ and \\ Mark A. Demitrack* \\ *Department of Psychiatry, University of Michigan Medical Center, Ann Arbor, Michigan, U.S.A.: \\ $\dagger$ Departments of Internal Medicine and of Microbiology and Immunology, University of Michigan Medical \\ Center, Ann Arbor, Michigan, U.S.A.; and †Department of Psychiatry, Istanbul Faculty of Medicine, \\ Istanbul, Turkey
}

(Received 2 September 1993; accepted I October 1993)

\begin{abstract}
Summary-The psychobiology of idiopathic fatigue has received renewed interest in the medical literature in recent years. In order to examine the relation between chronic, idiopathic fatigue and specific subtypes of depressive illness, we characterized the pattern and severity of scasonal symptom variation in 73 patients with chronic, idiopathic fatigue, compared to patients with major depression $(n=55)$, atypical depression $(n=35)$, and seasonal affective disorder $(n=16)$. Fifty of the fatigued subjects also met the specific Centers for Disease Control and Prevention case criteria for chronic fatigue syndrome, though this definition was unable to discriminate a distinct subgroup of patients, based on their seasonality scores alone. As a group, the fatigued subjects reported the lowest levels of symptom seasonality of any of the study groups. Further, even in those fatigued subjects with scores in the range of those seen in patients with seasonal affective disorder, seasonality was not reported to be a subjectively distressing problem. These findings lend support to the idea that although chronic fatigue shares some clinical features with certain mood disorders, they are not the same illnesses. These data are also consistent with the emerging view that chronic fatigue represents a heterogeneously determined clinical condition.
\end{abstract}

\section{Introduction}

THE PSYCHOBIOLOGY of idiopathic fatigue has received renewed interest in the medical literature in recent years. While the symptom of fatigue is a common accompaniment of various psychiatric illnesses, chronic idiopathic fatigue has also been proposed as a discrete clinical syndrome, often preciptated by an acute infectious illness and exacerbated by physical and emotional stressors. In 1988, the Centers for Disease Control and Prevention (CDC) formalized a working case definition of "chronic fatigue syndrome" (CFS) as the syndromal presentation of chronic, debilitating fatigue, accompanied by feverishness, and a variety of diffuse pains and other constitutional complaints (Holmes et al., 1988).

\footnotetext{
Author to whom correspondence should be addressed: Mark A. Demitrack, University of Michigan Medical Center, 1500 Eas1 Mcdical Center Drive, Ann Arbor, MI 48109-01 I6, U.S.A.

This work was supported in part by a bursary from The Ciba Foundation awarded to Dr Yargiç.
} 
Two principal theories have long dominated attempts at pathophysiologic explanations of this syndrome (Demitrack \& Greden, 1991a). One has focused on the behavioral antecedents of the illness, maintaining that the disorder represents the psychological aftermath of an acute infectious event in emotionally susceptible individuals. A number of recent studies employing structured psychiatric interviews in patients with unexplained, chronic fatigue would appear to support this view and have emphasized that formally diagnosable psychiatric illnesses are present in well over half of all cases, the most common diagnoses being the major mood disorders (Katon, Buchwald, Simon, Russo, \& Mease, 1991; Kruesi, Dove, \& Straus, 1989; Manu et al., 1989; Wessely \& Powell, 1989; Wood, Bentall, Gopfert, \& Edwards, 1991). However, it is often difficult to determine whether the psychiatric disorder is fully explanatory of the clinical presentation, a secondary manifestation of a primary disease process, or a coincident disease which modulates the presentation and course of the fatigue state. Indeed, it has recently been suggested that the specific requirement for multiple somatic symptoms in the CDC case definition may bias towards this overrepresentation of psychiatric illness (Katon \& Russo, 1992). Alternatively, it has been proposed that CFS represents a lasting and specific immune dysfunction induced by the infectious agent. Such a model was advanced, in part, as a result of initial reports of abnormal patterns of antibody responses to certain viral antigens, along with non-specific elevations in antinuclear antibodies and immune complexes in these patients (Jones et al., 1985; Straus et al., 1985; Tobi et al., 1982). Subsequent observations of disturbances in humoral and cellular immune mechanisms, such as immunoglobulin subclass deficiencies, defects in natural killer cell or other T-cell subset number and function, and abnormal levels of serum cytokines have further increased interest in this view (Calgiuri et al., 1987; Chao et al., 1990, 1991; Cheney, Dorman, \& Bell, 1989; Kilber, Lucas, Hicks, Poulos, \& Jones, 1985; Klimas, Salvato, Morgan, \& Fletcher, 1990; Komaroff, Geiger, \& Wormsely, 1988; Landay, Jessop, Lennette, \& Levy, 1991; Lloyd, Wakefield, Boughton, \& Dwyer, 1989; Straus et al., 1989, 1993; Tosato, Straus, Henle. Pike. \& Blaese, 1985) However, at the present time, the lack of a specific relation between any putative biological correlate of this syndrome and its clinical symptoms or course, coupled with methodologic difficulties, such as the absence of psychiatrically ill comparison groups or adequate behavioral descriptors of the fatigued individuals, have hampered a clearer interpretation of the data and a more reasoned appreciation of the relative contributions of either of these pathophysiologic mechanisms, or others, to the development of this syndrome.

In this report we characterize the seasonal symptom patterns of patients with chronic, idiopathic fatigue and provide comparisons to patients with a variety of typical and atypical mood disorders. We believe that this comparison may be useful for several reasons. Depression is a heterogeneous illness. Indeed, it has been suggested that a specific, biologically distinct subtype of depression may be characterized by a predominant disturbance in energy level, associated with a reversal of the typical vegetative features of depression, the so-called "atypical depression" (Quitkin et al., 1984). It has been noted that some paticnts with such an atypical depression report a significant seasonal variation to their symptoms, similar in magnitude to that seen in patients with seasonal affective disorder (SAD), suggesting that these illnesses may, therefore, be closely related clinical entities (Pande, Haskett, \& Greden, 1992). Likewise, we hypothesize that the presence of com- 
parable patterns of seasonal symptom variation in patients with CFS may suggest a close relation between this entity and certain "anergic" depressive illnesses. In this regard, we have recently shown that patients with CFS demonstrate impaired activation of the hypothalamic-pituitary-adrenal axis (Demitrack et al., 1991b). A recent study reported that this hormonal abnormality may also be seen in patients with $\mathrm{S} \Lambda \mathrm{D}$ during the depressed phase of their illness (Joseph-Vanderpool et al., 1991). Hence, a more specific examination of the variation in mood and related symptoms across the seasons may provide a useful point of comparison between patients with CFS and the depressive disorders.

\section{Subjects and Methods}

The Chronic Fatigue Clinic at the University of Michigan Medical Center was opened in May of 1990 for the evaluation of self- and physician-referred patients with chronic, unexplained fatigue. All patients referred to the clinic are evaluated first by a member of the Infectious Diseases staff. Upon initial visit, each patient undergoes a complete medical history and physical examination, which also includes a formal review of the CDC criteria for CFS (Holmes et al., 1988). Clinical laboratory testing, if not already recently performed elsewhere, includes a CBC with differential, serum electrolytes, liver function tests, glucose, creatinine, BUN, calcium, phosphorous, creatine phosphokinase, urinalysis, sedimentation rate, and thyroid function testing. Other laboratory testing, including specific infectious studies, is performed when symptoms or exposure history justify these tests clinically. If, after this initial cvaluation, underlying medical conditions are rulcd out, then the patient is scheduled for a return visit to evaluate further the nature and scope of their idiopathic complaints. At the follow-up visit, and prior to the initiation of treatment, a psychiatric interview is conducted to assess the presence of DSM-IIIR psychiatric diagnoses or accompanying psychiatric symptomatology. A standardized intake packet is completed by all patients. Included in this packet are dimensional measures of psychological distress including the Symptom Checklist-90, revised version (SCL-90R) (Derogatis, 1975), and the Seasonal Pattern Assessment Questionnaire (SPAQ) (Rosenthal, Bradt, \& Wehr, 1987). Subjects whose primary complaint of fatigue could be fully explained by medical or psychiatric diagnoses were not included in the present report. In the sample we report here, we follow the recent recommendations of the joint NIAID/NIMH workshop to assess systematically the psychiatric symptoms a priori at entry, and then, a posteriori, examining the relation between the psychiatric symptoms and the other dependent variables we report here (i.e., the measures of seasonal symptom variation) (Schluederberg, et al., 1992).

A total of 73 patients ( 55 females, 18 males) with chronic, idiopathic fatigue as the presenting complaint were recruited for the present study. On the basis of the medical and psychiatric assessment described above, 50 subjects met full criteria for the CDC case definition of CFS (fully syndromal), while 23 subjects had an insufficient number of minor criteria (subsyndromal), but were otherwise symptomatic.

Three separate populations of primarily mood-disordered patients were compared to the fatigued subjects: (a) patients diagnosed with DSM-IIIR Major Depressive Disorder (MDD, $n=55$ ) (APA 1987); (b) patients with Major Depressive Disorder who also met Columbia criteria for definite Atypical Depression (Quitkin et al., 1984) (AD, $n=35$ ); and 
(c) patients with Major Depressive Disorder who met criteria for seasonal affective disorder (Rosenthal et al., 1984) (SAD, $n=16$ ). The entire cohort of mood-disordered patients was recruited through the Depression Program of the Department of Psychiatry at the University of Michigan Medical Center, by senior psychiatrists blind to the purpose of this study.

Results on the SPAQ in the four patient groups were scored as previously described (Kasper, Wehr, Bartko, Gaist, \& Rosenthal, 1989). Briefly, this instrument inquires about seasonal changes in several functional domains, including sleep length, activity, mood, appetite, weight, and energy level. The items are rated from 0 (no scasonal change) to 4 (extreme seasonal change). A global seasonality score, ranging from 0 to 24 , is generated based on the average scores of the individual items. A further set of questions determines the reactivity to various environmental or weather changes, and the seasonal pattern of the symptoms, with winter patterns defined as feeling worst in January and/or February (with or without any other affected months), and summer patterns as feeling worst in July and or August (with or without other affected months). The overall perceived severity of the seasonal changes is also elicited.

The questionnaires were administered to all subjects in an outpatient setting over the course of the year, for all patient groups, except for those with SAD, who completed the SPAQ only during the fall/winter months. Twenty-nine $(39 \%)$ of the fatigued subjects completed the SPAQ during fall/winter, while $36(65 \%)$ and $24(68 \%)$ of MDD and AD patients, respectively, responded during that season. We therefore examined for the possible influence of scason at the time of completion of the SPAQ by comparing seasonality scores obtained during the spring and summer with those obtained during the fall and winter.

Statistical comparisons between groups were conducted using contingency tables for noncontinuous variables, while analysis of variance (ANOVA) was employed for continuous variables. When the ANOVA showed a significant main effect, post-hoc analyses were performed using two-lailed Mann-Whitney U-tests between diagnostic groups. Other tests used are indicated in the text. Significance levels were adjusted for multiple comparisons using the Bonferroni correction. Results are expressed, where relevant, as cither the mean 11 $S D$ or the median.

\section{Results}

Women outnumbered men in all groups by at least three to one. Subjects were most often single in the atypically depressed group. Otherwise, no differences in age, marital status or educational level were detected between groups. Since there were no differences between the subsyndromal and fully syndromal patients with chronic fatigue on any of the measures reported here, their results are combined for purposes of statistical analysis. The season during which the fatigued subjects, MDD or AD patients completed the SPAQ did not appear to influence the results; no differences in seasonality scores were observed between spring/summer and fall/winter responses (two-tailed, unpaired $t$-test, data not shown).

\section{Seasonal symptom pattern and severity}

The mean global seasonality score for the overall group of fatigued patients was $6.6 \pm 5.7$, which was significantly lower than the three depressed comparison groups (Table 1). Fifty- 
seven fatigued subjects reported no or little seasonal variation in their symptoms, while 16 $(22 \%)$ reported a global seasonality score greater than or equal to 10 , a cutoff score previously found to indicate clinically significant seasonal symptom change (Kasper et al., 1989). In contrast, a higher proportion of the three affectively ill patient groups reported a global scasonality scorc greater than or equal to $10(47,51$ and $100 \%$ for the MDD, AD and SAD groups, respectively).

Table 1

Seasonal Pattern Assessment Questionnaire (SPAQ) Scores of Patients with Chronic Fatigue, and Patients with Major Depression, Atypical Depression, and Seasonal Affective Disorder

\begin{tabular}{|c|c|c|c|c|c|c|}
\hline & Fatigue & MDD & $\mathrm{AD}$ & SAD & $F$ ratio & $p$ \\
\hline \multicolumn{7}{|l|}{ Seasonality scores } \\
\hline Sleep length & $0.93 \pm 1.2$ & $1.34 \pm 1.2^{\mathrm{a}}$ & $1.31+1.3$ & $2.75 \pm 0.8^{\mathrm{d}}$ & 10.66 & .0001 \\
\hline Social activity & $1.26 \pm 1.2$ & $1.64 \pm 1.1$ & $1.86 \pm 1.3$ & $3.12 \pm 0.8^{\mathrm{d}}$ & 11.27 & .0001 \\
\hline Mood & $1.47 \pm 1.3$ & $1.75 \pm 1.2$ & $1.89 \pm 1.2$ & $3.63 \pm 0.5^{\mathrm{d}}$ & 13.75 & .0001 \\
\hline Weight & $0.97 \pm 1.2$ & $1.15 \pm 1.0$ & $1.40 \pm 1.1$ & $2.44 \pm 1.1^{\mathrm{d}}$ & 7.69 & .0001 \\
\hline Appetite & $0.82 \pm 1.0$ & $1.20 \pm 1.2$ & $1.29 \pm 1.2$ & $2.75 \pm 0.7^{\mathrm{d}}$ & 13.64 & .0001 \\
\hline Energy & $1.25 \pm 1.3$ & $1.65 \pm 1.4$ & $1.86 \pm 1.2^{\mathrm{a}}$ & $3.44 \pm 0.5^{\mathrm{d}}$ & 13.70 & .0001 \\
\hline Global & $6.66 \pm 5.7$ & $8.73 \pm 6.0^{a}$ & $9.60 \pm 5.9^{\mathrm{a}}$ & $18.10 \pm 2.7^{\mathrm{d}}$ & 18.13 & .0001 \\
\hline \multicolumn{7}{|l|}{ Perceived } \\
\hline Severity $(0-4)$ & $0.78 \pm 1.4$ & $1.33 \pm 1.6^{\mathrm{a}}$ & $1.54 \pm 1.5^{\mathrm{b}}$ & $4.00 \pm 0.8^{d}$ & 21.63 & .0001 \\
\hline Weight $\Delta$ (lbs) & $2.2 \pm 1.4$ & $2.5 \pm 1.2$ & $3.2 \pm 1.5^{\mathrm{b}}$ & $3.5 \pm 1.2^{\mathrm{c}}$ & 6.46 & .0004 \\
\hline \multicolumn{7}{|l|}{ Hours Slept daily } \\
\hline Winter & $9.8 \pm 2.4$ & $8.1 \pm 2.1^{\mathrm{d}}$ & $9.4 \pm 2.2$ & $9.4 \pm 1.9$ & 5.74 & .0001 \\
\hline Spring & $9.1 \pm 2.4$ & $7.3 \pm 1.2^{\mathrm{d}}$ & $8.1 \pm 1.4$ & $7.3 \pm 1.3^{b}$ & 10.53 & $.000 \mathrm{l}$ \\
\hline Summer & $8.9 \pm 2.1$ & $7.3 \pm 1.5^{\mathrm{d}}$ & $7.8 \pm 1.5^{\mathrm{a}}$ & $7.0 \pm 0.9^{c}$ & 9.96 & .0001 \\
\hline Fall & $9.5 \pm 2.4$ & $7.5 \pm 1.5^{\mathrm{d}}$ & $8.6 \pm 1.8$ & $8.8 \pm 1.7$ & 9.11 & .0001 \\
\hline
\end{tabular}

Note. Values are expressed as the mean $\pm 1 S D$. Weight change $(\Delta)$ is the reported weight fluctuation between the seasons, in pounds. Post-hoc paired comparisons using the two-tailed Mann-Whitney U-test were performed using the combined fatigue group as the reference, ${ }^{\mathrm{a}} p<.05,{ }^{\mathrm{b}} p<.01,{ }^{\mathrm{c}} p<.001,{ }^{\mathrm{d}} p<.0001$.

Sleep length during the different seasons was not significantly different among the AD, SAD or fatigued cohorts, with the exception of sleep length during the summer months, when SAD patients showed a significant reduction in the number of hours slept compared to patients with chronic fatigue (Table 1). Interestingly, the MDD group reported shorter sleep periods than the fatigued subjects throughout the year (Table 1). Weight change was also more variable in the AD and SAD groups than in the chronic fatigue or MDD patient samples.

In all patient groups, when seasonal variation in symptoms was apparent, a winter pattern predominated. In general, the fatigued subjects subjectively considered seasonality of symptoms to represent no or only a mild problem, compared to the perception of their mooddisordered counterparts (Table 1). Weather or environmental sensitivity was endorsed by a low number of fatigued subjects, with a frequency of item response ranging from 3 to $57 \%$, with the lowest response rate being noted for 'sunny' and 'dry' days ( $3 \%$ each), and the highest for 'humid' and 'foggy' days ( 57 and $43 \%$, respectively). 
Comparison of highly seasonal fatigued patients with low seasonal fatigued patients and patients with $S A D$

To explore the possibility that those chronically fatigued patients who reported a high global seasonality score also presented higher degrees of psychopathology in general, we compared the behavioral characteristics of this highly seasonal chronic fatigue group to the less seasonal fatigued subjects. Indeed, the 16 fatigued subjects who reported substantial degrees of seasonal symptom change reported generally higher scores on the SCL-90R than the less seasonal fatigue patients, though these differences reached statistical significance (after correction for multiple comparisons) only for the obsessive-compulsive and phobic anxiety factor scores (Table 2). There was no difference in the percentage of subjects who met the full CDC case definition for CFS in the highly seasonal fatigue group compared to their less seasonal counterparts (Fisher's exact test, ns).

Table 2

Comparison of SCL-90R Scores in High and Iow Seasonal Chronic Fatigue Patients

\begin{tabular}{|c|c|c|c|c|}
\hline & $\begin{array}{l}\text { Low seasonality } \\
\qquad(\mathrm{CSS}<10)\end{array}$ & $\begin{array}{l}\text { High seasonality } \\
\quad(\text { GSS } \geqslant 10)\end{array}$ & = score & $p$ value \\
\hline \multicolumn{5}{|l|}{ SCL-90-R subscale score } \\
\hline Somatization & $1.4 \pm 0.7$ & $1.7 \pm 0.8$ & 1.46 & ns \\
\hline Obsessive-compulsive & $1.4 \pm 0.8$ & $2.1 \pm 0.9$ & 2.92 & $<\left..0\right|^{*}$ \\
\hline Interpersonal sensitivity & $0.7 \pm 0.7$ & $1.2 \pm 1.0$ & 2.05 & $<.05$ \\
\hline Depression & $1.3 \pm 0.9$ & $1.7 \pm 0.9$ & 1.87 & ns \\
\hline Anxicty & $0.8 \pm 0.8$ & $1.2 \pm 0.9$ & 2.57 & $<.01$ \\
\hline Hostility & $0.6 \pm 0.6$ & $1.1 \pm 1.1$ & 1.98 & $<.05$ \\
\hline Phobic anxicty & $0.3 \pm 0.4$ & $1.0 \pm 1.0$ & 3.93 & $<.001^{*}$ \\
\hline Paranoid idcation & $0.5 \pm 0.6$ & $1.0 \pm 1.0$ & 2.81 & $<01$ \\
\hline Psychoticism & $0.5 \pm 0.4$ & $0.8 \pm 0.9$ & 2.20 & ns \\
\hline Global Symptom Index & $0.9 \pm 0.6$ & $1.4 \pm 0.8$ & 2.61 & $<.01$ \\
\hline
\end{tabular}

Note. Values are expressed as the mean \pm 1 SD. Statistical comparisons were performed via 1wo-tailed MannWhitney U-test: * indicates significance remains after Bonferroni correction for multiple comparisons.

Despite the similarity in seasonal symptom variation between highly seasonal fatigued subjects and patients with SAD, the highly seasonal chronic fatigue patients generally perceived that seasonality, per se, was at worst only a mild contributor to their overall distress (data not shown), in this way differing from reports of SAD patients.

\section{Discussion}

Patients with idiopathic, chronic fatigue report seasonal variation in symptoms which is similar in pattern and magnitude to that seen in non-clinical populations (Hardin et al.. 1991; Kasper et al., 1989), and differs significantly from that seen in patients with a variety of mood disorders (Hardin et al., 1991). Moreover, even when the degree of seasonal variation is prominent, patients with chronic fatigue do not, as a group, report that these changes are an essential feature of their illness. It is of interest that none of the measures reported here was able to distinguish chronically fatigued subjects who met the full CDC case definition for CFS from those who fell short of this definition duc to an insufficient number of minor criteria. This observation questions the ability of the current CDC case 
definition to discriminate a unique patient group, different from the larger population of chronically fatigued patients. Hence, subsyndromal chronic fatigue patients exist on a continuum, and may therefore represent a less symptomatic, but otherwise pathophysiologically similar group to the fully syndromal CFS patients. Alternatively, it is possible that the patient population described here differs from that of other studies. The utilization of a specialty fatigue clinic within a tertiary care referral center, and the careful screening of patients by both internal medicine and psychiatry staff may have biased our subject selection towards a more severely ill, and perhaps more homogeneous, patient group.

Several observations of the data we present deserve comment, and may serve to highlight areas for future study. As noted above, patients with chronic, idiopathic fatigue report apparently normal patterns of seasonal symptom variation. Patients with MDD have also been reported to show degrees of seasonality not different from healthy individuals (Hardin et al., 1991). Interestingly, while the degree of seasonal symptom variation was closest to that of MDD patients, the reported number of hours slept daily across seasons by the fatigued patients was significantly different from MDD subjects, and more closely resembled the pattern seen in patients with $\mathrm{AD}$, or to SAD subjects during the winter. In other words, the pattern and severity of seasonal symptom change shows overlapping characteristics among the fatigued patients and each of the different depressed groups, nevertheless, chronically fatigued subjects cannot be fully subsumed under any of these categorical definitions on this parameter alone. These findings lend support to the idea that although chronic fatigue shares some clinical features with certain mood disorders, they are not the same illnesses. Indeed, the data reported here are consistent with the view that chronic fatigue represents a heterogeneously determined clinical condition, rather than a discrete disease with a singular cause.

Twenty-two percent of the chronic fatigue patients we describe reported highly seasonal variation to their symptoms. Although this figure is similar to the proportion of highly seasonal subjects seen in non-clinical community samples (Kasper et al., 1989), the specific characteristics of this subgroup of fatigued patients raise several considerations regarding the relation between SAD and chronic fatigue states, and bear on a more complete understanding of the nature of chronic fatigue. First, it may be possible that the highly seasonal fatigued patients represent misdiagnosed patients with SAD. If true, this is a clinically meaningful issue, since the treatment options for chronic idiopathic fatigue are few, and the identification of a specifically treatment-responsive subset of fatigued patients would be of immense clinical interest. In this regard, the recent report of two cases of SAD presenting as CFS speaks to this point (Lam, 1991). Interestingly, in that report, the seasonal symptom variation was not recognized by those two patients, similar to the perception of the paticnts in the chronic fatigue sample we describe here. As further support for the potential identity between SAD and at least a subset of idiopathic fatigue states, it is of note that patients with SAD have been reported to demonstrate relatively impaired reactivity of the hypothalamicpituitary-adrenal axis during the depressed phase of their illness (Joseph-Vanderpool et al., 1991), similar to the neuroendocrine pattern we have previously reported in patients with CFS (Demitrack et al., 1991b). Alternatively, SAD may be one of the many psychiatric disorders which appears to associate with chronic fatigue states, and hence may be a cause 
or a consequence of the fatigue state. Or, the presence of SAD may merely be a coincidental illness, pathophysiologically unrelated to the primary disease process producing the fatigue.

Most studies employing formal structured psychiatric interview techniques report that somatization disorder is present in approximately $10-15 \%$ of most populations with CFS, an incidence much higher than that seen in community samples. While the reason for this observation is unclear, it has been proposed that the propensity towards somatization in this patient group may represent a desire to adopt an illness model which posits an external source to the disease process (e.g., a virus). Such a psychological position may serve an adaptive purpose, protecting the individual from the sense of guilt and shame associated with identifying the disease process as having internal psychological determinants. Considered in this light, the responses of the fatigued patients to the SPAQ may be due in large part to this tendency to somatize, and hence to manifest a bias merely to respond positively to questions about symptoms. If this model is correct, light therapy would be ineffective in such patients, or only produce a partial resolution of symptoms (e.g., improvement in mood, concentration and energy level, but little change in sore throat, myalgias, lymphadenopathy and other physical complaints).

It is of interest that the subgroup of fatigued subjects with high seasonal symptom variation also showed moderately higher degrees of self-reported psychopathology, most notably anxiety symptoms. Hence, it is possible that this subgroup of chronically fatigued patients, charactcrized by significant scasonal symptom variation, represents a distinct psychopathological entity, with characteristics similar to patients with classical SAD. Future studies appear warranted. For example, further subclassification of chronic fatigue subjects into those with or without anxiety and/or depressive symptoms, may aid in limiting the heterogeneity of the group. In so doing, subsequent comparisons of clinical and biological characteristics may yield clearer definitions of CFS boundaries. Additionally, the study of subgroup responses to specific interventions (i.e., light therapy) would be particularly informative, and may aid in the more reasoned diagnostic formulation and treatment of this complex group of patients.

\section{References}

American Psychiatric Association (1987). Diagnostic and statistical manual of mental disordew. 3rd ed. rev. Washington, DC: Author

Calgiuri, M., Murray. C., Buchwald, D., Levine, H., Cheney, P., Peterson, D., Komarof, A. I.., \& Ritz, J. (1975). Phenotypic and functional deficiency of natural killer cells in patients with chronic fatigue syndrome. Journal of Immunology, 139, 33063313.

Chao, C. C.. Gallagher, M., Phair, J. \& Peterson. P. K. (1990). Serum neopterin and interleukin-6 levels in chronic fatigue syndrome. Journal of Infectious Diseases, 162, 14121413.

Chao, C. C., Janoff, E. N., Hu, S., Thomas, K.. (Gallagher, M., Tsang, M. \& Petcrson, P. K. (1991). Altered cytokine release in peripheral blood mononuclear cell cultures from patients with the chronic latigue syndrome. Crtokine, 3, 292298.

Cheney, P. R., Dorman, S. E. \& Bell, D. S. (1989). Interleukin-2 and the chronic fattgue syndrome. Amals of Intrinal Modicinc, $110,321$.

Demitrack, M. A., \& Greden, J. F. (199lia). Chronic fatigue syndrome: The need for an inlegrative approach. Biological Psychiatry, 30, 747752.

Demitrack, M. A.. Dale, J. K.. Straus, S. E., Laue, I.. Listwak, S. J., Kruesi, M. J. P., Chrousos. G. P.. \& Gokd. P. W. (1991b). Evidence for impaired activation of the hypothlamic pituitary adrenal axis in patients with chronic tatigue syndrome. Journal of Clinical Endorinologl and Metabolism, 73, 12241234.

Derogatis, L. R. (1975). The SCL-90-R. Baltimore: Clinical Psychometrics Research. 
Hardin, T., Wehr, T., Brewerton, T., Kasper, S., Berretini, W., Rabkin, J., \& Rosenthal, N. (1991). Evaluation of seasonality in six clinical populations and two normal populations. Journal of Psychiatric Research, 25, 7587 .

Holmes, G., Kaplan, J., Gantz, N., Komaroff, A. L., Schonberger, L. B., Straus, S. E., Jones, J. F., DuBois, R. E., Cunningham-Rundles, C., Pahwa, S., Tosato, G., Zegans, L. S., Purtilo, D. T., Brown, N., Schooley, R. T. \& Brus, I. (1988). Chronic fatigue syndrome: A working case definition. Annals of Internal Medicine, 108, 387 389.

Jones, J. F., Ray, G., Minnich, L. L., Hicks, M. J., Kibler, R., \& Lucas, D. O. (1985). Evidence for active EpsteinBarr virus infection in patients with persistent, unexplained illnesses: Elevated anti-early antigen antibodies. Annals of Internal Medicine, 102(1), 17.

Joseph-Vanderpool, J. R., Rosenthal, N. E., Chrousos, G. P., Wehr, T. A., Skwerer, R., Kasper, S., \& Gold, P. W. (1991). Abnormal pituitary-adrenal Responses to CRH in patients with seasonal affective disorder: Clinical and pathophysiological implications. Journal of Clinical Endocrinology and Metabolism, 72(6), 1382 1387.

Kasper, S., Wehr, T., Bartko, J., Gaist, P., \& Rosenthal, N. (1989) Epidemiological findings of seasonal changes in mood and behavior. Archives of General Psychiatry, 46, 823-833.

Katon, W. J., Buchwald, D. S., Simon, G. E., Russo, J. E., \& Mease, P. J. (1991). Psychiatric illness in patients with chronic fatigue and those with rheumatoid arthritis. Journal of General Internal Medicine, 6, $277-285$.

Katon, W., \& Russo, J. (1992). Chronic fatigue syndrome: A critique of the requirement for multiple physical complaints. Annals of Internal Medicine, 152, $1604-1609$.

Kibler, R., Lucas, D. O., Hicks, M. J., Poulos, B. T., \& Jones, J. F. ( I985). Innmune function in chronic active Epstein-Barr virus infection. Journal of Clinical Immunology, 5, $46 \cdots 54$.

Klimas, N. G., Salvato, F. R., Morgan, R., \& Fletcher, M. A. (1990). Immunological Abnormalities in Chronic Fatigue Syndrome. Journal of Clinical Microbiology, 28, 1403-1410.

Komaroff, A. L., Geiger, A. M., \& Wormsely, S. (1988). IgG subclass deficiencies in chronic fatigue syndrome. Lancet, 1, 12881289.

Kruesi, M. J. P., Dale, J., \& Straus, S. E. (1989). Psychiatric diagnoses in patients with the chronic fatiguc syndrome. Journal of Clinical Psychiatry, 50, 53-56.

Lam. R. W. (1991). Seasonal affective disorder presenting as chronic fatigue syndrome. Canadian Journal of Psychiatry, 36, 680-682.

Landay, A. L., Jessop, C., Lennette, E. T., \& Levy, J. A. (1991). Chronic fatigue syndrome: Clinical condition associated with immune activation. Lancet. 338(8769), 707-712.

Lloyd, A. R., Wakefield, D., Boughton, C. R., \& Dwyer, J. M. (1989). Immunological abnormalities in the chronic fatigue syndrome. Medical Journal of Australia, 151, 122-124.

Manu, P.. Matthews, D., Lane, T., Tennen, H., Hesselbrock, V., Mendola, R., \& Affleck, G. (1989). Depression among patients with a chief complaints of chronic fatigue. Journal of Affective Disorders, 17, $165-172$.

Pande, A., llaskett, R., \& Greden, J. (1992). Seasonality in atypical depression. Bioloyical Psychiatry, 31, 965 967.

Quitkin, F., Harrison, W., Liebowitz, M., McGrath, P., Rabkin, J. G., Stewart, J., \& Markowitz, J. (1984). Defining the boundaries of atypical depression. Journal of Clinical Psychiatry, 45, 19-21.

Rosenthal, N. E., Sack, D. A., Gillin, J. C., Lewy, A. J., Goodwin, F. K., Davenport, Y., Mueller, P. S., \& Wehr, T. A. (1984). Seasonal affective disorder. A description of the syndrome and preliminary findings with light therapy. Archives of General Psychiatry, 41, 7280.

Rosenthal, N. E., Bradt, G. H., \& Wehr, T. A. (1987). Seasonal pattern assessment questionnaire (SPAQ), Bethesda: Clinical Psychobiology Branch, NIMH.

Schluederberg. A., Straus, S. E., Peterson, P., Blumenthal, S., Komaroff, A. L., Spring, S. B., Landay, A., \& Buchwald, D. (1992). Chronic fatigue syndrome research: Definition and medical outcome assessment. Annals of Internal Medicine, $117(4), 325 \cdots 331$.

Straus, S. E., Tosato, G., Armstrong, G.. Lawley, T., Preble, O. T., Henle, W., Davey, R., Pcarson, G., Epstein, J., \& Brus, I. (1985). Persisting illness and fatigue in adults with cvidence of Epstein-Barr virus infection. Annals of Internal Medicine, 102(1), 7 16.

Straus, S. E., Dale, J. K., Peter, J. B., \& Dinarello, C. A. (1989). Circulating lymphokine levels in the chronic fatigue syndrome. Journal of Infectious Diseases, 160, $1085 \cdot 1086$.

Straus, S. E., Fritz, S., Dale, J., Gould, B., \& Strober, W. ( 1993). Lymphocyte phenotype analysis suggests chronic immune stimulation in patients with chronic fatigue syndrome. Journal of Clinical Immunology, 13(I), 30-40.

Tobi, M., Morag, A., Ravid, 7., Showers, I., Feldman-Weiss, V., Michaeli, Y., Ben-Chetrit, E., Shalit, M., \& Knobler. H. (1982). Prolonged atypical illness associated with serological evidence of persistent Epstein-Barr virus infection. Lancet, 9, 61-64.

Tosato, G., Straus, S. E., Henle, W., Pike, S. E., \& Blaese, R. M. (1985). Characteristic T-cell dysfunction 
in patients with chronic active Epstein-Barr virus infection (chronic infectious mononucleosis). Journal of Immunology, 134, 3082 3088.

Wessely, S., \& Powell, R. (1989). Fatigue syndromes: A comparison of chronic "postviral" fatigue with neuromuscular and affective disorders. Journal of Neurology, Neurosurgery, and Psychiatry, 52, $940-948$.

Wood, G. C., Bentall, R. P., Gopfert, M., \& Edwards, R. H. (1991). A comparative psychiatric assessment of patients with chronic fatigue syndrome and muscle disease. Psychological Medicine, 21, 619-628. 